\title{
On Mental Concepts and Physical Concepts
}

\author{
Donald D. Davidson
}

Stanford University

It is a very great pleasure and privilege for me to talk to this distinguished group. I have already profited a good deal from listening to the speakers and questioners, and I have had a hard time deciding how I could add anything appropriate of my own. I have had two thoughts about this. A number of you observed that the most philosophical member of this symposium is a distinguished physicist and I have no hope at all of competing with him as a philosopher, much less as a physicist. But I do not see why that should not stop me from trying -trying is one of those special activities reserved for rational agents-and so, as a rational agent, I am going to try to be as metaphysical as Professor Bose. And my second thought is that what we would like to accomplish here is some kind of meeting of minds, both a meeting of experts in different subjects, and also a meeting of representatives of different cultures.

I think that there are different ways in which minds can be brought into contact with each other. One of them is to try to achieve a maximum of agreement; the other is by, shall I say, bringing them into contact forcibly, knocking them together, so to speak, and I think it suits my personality a good deal better to attempt the second of these, but I have high hopes that the things I am going to say will meet with practically universal disagreement.

I am going to address myself to the third topic on the announced list of topics for discussion of this symposium. That topic began with a question, which was something like this, "Can we hope to give an exhaustive description of man and his behaviour, in the physical vocabulary or concepts of the physical sciences-, that is, the sciences which treat of non-animal, non-human phenomena on a par with others?" And the third topic ends with what may sound like a rather strange thing. It says, "We wish to make clear where we stand."

Well, do we have any choice, but to stand where the truth is? That is, it seems strange to say 'Is snow white, let us take a stand'. But yet I believe that there is something quite appropriate in the idea of taking a stand in this matter. There is an element of choice involved in the answer to this question, in my opinion.

Philosophers and others have always been worried that if it should turn out that an exhaustive description of man and his actions can be given in the physical sciences, that this would eliminate, make incomprensible, the notion of freedom of action, and that as a consequence, we could not apply moral considerations of praise and blame to human actions at all. Kant, for example, phrased the puzzle by saying, "If rather", Kant said, "Since physical determinism in a broad and perhaps 
rather vague sense, since physical determinism holds for all of nature, therefore man,-how is freedom possible?" The standard answer to this question in the empiricist and analytic tradition of the West has always been that there is no conflict at all between determinism and free will, that it is only due to a confusion of determinism with compulsion, that any one has ever worried that determinism would hinder, interfere with, or be relevant to, the freedom of human action. Kant, however, was not satisfied with this answer, and I believe myself that though this answer is correct so far as it goes, it is radically incomplete. I shall follow Kant in connecting the question with which we are dealing directly with the traditional problem of the relation of mind and material, thus re-uniting two problems which in recent discussion have been divorced, and I shall also follow Kant in interpreting the realm of the mental as including behaviour, that is, action. It is a mistake, in my opinion, to suppose that the mark of the mental is that it is immaterial, private or subjective. The mark of the mental is rather intentionality, and this is something-intentionality is something-which is exhibited by a certain kind of material objects, namely persons.

I begin by vaguely characterizing two realms of concepts which I shall call P-concepts for physical concepts, and M-concepts for mental concepts. Under Mconcepts, I include the notion of intentional action of motive, belief, desire, attitude and a very great many more besides. All of these are connected in an essential way with the notion of intention, purpose, and of human action. I have a harder time giving a list of P-concepts, because P-concepts apply to the same phenomena as the M-concepts as well as to others besides. But it may be some help at least in understanding the things that I will say, if I say that one important metaphysical characteristic of what I call physical concepts is that they are tied to the common sense notion of a physical object which has a location in space and time, which undergoes changes which are physical events, whose changes are governed by laws, that is, physical laws. In order to forestal misunderstanding, I would like to say that I believe the concepts of physical object, of physical event as a change in a physical object or physical situation, and the concepts of a physical law are completely tied up with one another. It would be quite misleading to say, first we know what physical objects are, and then we notice what changes they undergo, and then we discover, if we can, whether these changes are governed by laws. I would say rather a physical object is an object whose changes are governed by laws and this is something which is a priori, as Kant said. After all we must mean something by calling something a physical object and I would say this is a very rough sketch of part of what we do mean by calling something a physical object. Of course, this does not mean that we $a$ priori know what the laws are, which govern physical objects, but what we know a priori is only that something is not a physical object unless it is governed by some laws.

What is the relationship between these two sets of concepts? The first thing to realize is that the fact of a duality of concepts does not imply a duality of entities. That is, though we have both mental and physical concepts, they do not have to be two kinds of objects, two kinds of events, two kinds of changes, 
two kinds of states; all that we are committed to is that there are two ways of describing some objects, some states, some events; in other words, duality of concepts does not mean ontological duality.

I shall not argue for this view but simply announce my adherence to it. I believe myself that there is only one set of entities in the world. All of them can be described under the physical concepts, a few of them can be described under the mental concepts. But I want to make a very strong distinction between ontological dualism, which I am rejecting, and definitional dualism which, I believe, we must accept. The reason that we must accept definitional dualism is that I believe it is the case that we cannot give a satisfactory definition of any mental concept purely in terms of P-concepts. The evidence that no mental concept can be defined in terms of P-concepts is, I think, incomplete-it amounts to less than a proof-but the evidence is so overwhelming that it has persuaded just about every philosopher of science in the Western world. I will just mention two areas within which the evidence lies. There is, first of all, semantical evidence.

The sentences about belief, and hence about intention, and about hopes, wishes, desires, and so forth, seem absolutely to resist analysis in terms of purely extensional sentences. This fact, which was well known in the Middle Ages, was re-discovered by Frege who called all of those sentences which used mental concepts "oblique", (or said that they created "oblique") contexts because of the peculiar semantical properties which such sentences exhibited.

The second area, within which the evidence for non-translatability or nondefinitional reduction can be found, lies simply in a study of the failure of all particular attempts at analysing, for example, what it means to say that "A man is angry" in terms of neutrally described behaviour, whether actual or potential, or attempts to analyse the notion of meaning, or being a sign, in terms of purely extensional notions. Each of these attempts ends in failure and a study of the nature of the failures has persuaded a great many philosophers, --I will just mention Quine, Hempel, Feigl, Popper, Chisholm and every one except Carnap-has convinced them that there is a pattern in the failures, so that it is no accident that we cannot accomplish definitional reduction.

The view I put forward so far seems to me to be identical with the view recently proposed by Feigl in the second volume of The Minnesota Studies in the Philosophy of Science and also a view of a more limited sort put forward by J. J. C. Smart, in an article called "Sensations and Brain Processes". But I believe that every one who has reached the point that I have just arrived at, has gone on to assume that though you cannot define M-concepts in terms of P-concepts, there must be true laws or correspondences between M-states and P-states. That is, given any mental concept, such as a motive, desire, or a belief,-there will be a physical concept which will cover exactly those mental states; that is, given the P-concepts, one can define extensionally a class, and that class will be just the class covered by the mental concept. These two ways of defining the same class will not be synonymous, will not be logically equivalent, but they will be extensionally equivalent. And the most important point that I have to make is 
that I do not believe that this can be done either.

of course, if we consider any finite set of examples of mental states or events, there must trivially, on my theory, be a physical description which will cover just those. But in fact, of course, what we want as a law of correspondence is not an accidental correspondence, but one which, we have reason to believe, well apply to cases which we have not yet examined. And this is what I deny that we shall ever find.

As an analogy, I suggest that you consider the relationship between the property of goodness and the physical properties upon which, in any individual case, the goodness of a thing or action depends. As Moore pointed out long ago, it is absurd to say that two objects are exactly alike in all their physical properties, but one is good and the other is not. The property of being good is supervenient on physical properties in this sense that the goodness wholly depends upon the physical properties. But this does not mean that we can define the notion of being good on the basis of physical properties, nor even that we can give some physical property, no matter how complex, which applys to the good things and only to the good things. In my opinion it is no coincidence that goodness and intentionality are, both of them, supervenient on P-concepts, for goodness and intentionality are closely related to one another. Moral evaluations are in the first instant applied to intentional actions.

It follows from the things I have said, that is, if you agree-which I do not expect-with what I have said so far that there can be no true laws connecting P-concepts and M-concepts. That is, there are no laws that say, if a certain physical description holds, then everytime the corresponding mental description holds, or the corresponding mental state result. And there are, likewise, no laws which say everytime there is a mental state of this kind, there will be a physical state of that kind. In at least one sense, in which people have spoken of psychology, I am arguing, as philosophers will on a priori grounds, that psychology and the social sciences are impossible.

Nothing I have said shows that there cannot be M-M-laws,-laws connecting mental descriptions with mental descriptions, and indeed, I believe that there are legitimate branches of psychology which fall under this description, which connect intentional actions with intentional actions. Decision theory is an example of a branch of psychology which is in this respect one that has M-M-laws. However, for reasons which I shall not expand on now, I believe that the range of such sciences must be very limited and the reason is that the M-states do not constitute a closed system. Mental events are everywhere impinged upon by events which have no mental description at all, and, in consequence, there is no chance of having a highly developed and relatively deterministic science which consists just of MM-laws. There is no danger at all on the basis of these of having a genuine science for predicting in detail human intentional actions.

Nothing I have said implies that we cannot give causal scientific explanations of particular human actions, thoughts and the like. These negative remarks concern the possibility of laws, but if we choose a particular event, there is no reason why we cannot say this particular event (and here we describe it with a physical 
description) is a causal factor in the production of this mental event. So remember that that mental event also has a physical description and there may be a law that connects these two events, one of which we described in physical language, one of which we described in mental language, may connect those two events in a perfectly lawful way. So there is no reason, if you accept what I have said, to suppose that we cannot name the causes of mental phenomena, we cannot name them in particular cases, and no doubt, in classes of specially selected cases. What we cannot do is to produce really general laws, which connect events under physical descriptions with events under mental descriptions.

In general, when we state the cause of an event the descriptions that we use of cause and effect are not the descriptions which are relevant to the laws which we suppose connect those events. For example, if I drop this piece of chalk and it breaks, I am quite sure that the cause of the chalk breaking was that I dropped it. But there is no law that says whenever somebody drops a piece of chalk, it breaks. Because, of course, pieces of chalk often do not break when they are dropped.

The relevant laws have to do with the microscopic properties of this particular piece of chalk, about which I know nothing. I cannot say what the relevant laws are, that governed the breaking of this particular piece of chalk. And certainly the description "I drop this chalk", the description "This chalk break", are not at all the descriptions which would be needed in a scientific account which would lead to predictions. In exactly the same way, I am saying, we can give the causes of intentional actions often in physical terms. But we do not know what the laws are, which connect cause and effect, at least in general, because those laws do not employ the mentalistic concepts which we are interested in employing in talking about human action.

The network of mental concepts and the network of physical concepts are not like two alternative schemes where we can pass in some systematic way from one scheme to the other. There is no formula for doing this. If I may hint why this is the case, I say the application of mental concepts is governed by a different set of purposes, is anchored to a different weighting of evidence than the physical concepts. When we apply mental concepts, we must, logically must, enter in certain ways into the point of view, of the rational agent. We can significantly apply mental concepts only when there exist institutions of language, morality and law; and we can significantly attribute them only to objects whose actions we are prepared to evaluate morally and to whom we attribute rationality. These are necessary conditions for the application, and meaningful application, of mental concepts. While these are necessary conditions, there is nothing that forces us to apply mentalistic concepts. So we have at our disposal another set of concepts, the physical concepts which apply to just the same events and objects and states and which in a very important respect, is a superior set of concepts, for it is all inclusive and lends itself, or promises to lend itself, to far more accurate prediction. It is, therefore, partly an empirical question where and when we can meaningfully apply mental concepts, but it is also partly a matter for our choice. We can choose to treat men under physical concepts, if we wish, and of course, we 
must, if we want to study certain things about them. I am even prepared to suppose that if we build societies of machines, with institutions like language, and so on that we could meaningfully apply mentalistic concepts to such societies of machines. I confess that this does not worry me at all. The only thing that worries me is that at some point we should stop applying concepts of rationality, intentionality and moral evaluation to men. 